\title{
Photoinduced Phenomena in 6,6'-Dibromoindigo (Tyrian Purple): a Theoretical Study
}

\author{
(D) Vassil B. Delchev
}

Department of Physical Chemistry, University of Plovdiv, 4000 Plovdiv, Bulgaria

Author's e-mail address: vdelchev@uni-plovdiv.net

RECEIVED: January 12, 2021 * REVISED: March 15, 2021 * ACCEPTED: March 15, 2021

Abstract: The compound 6,6'-dibromoindigo, which is the main component of the pigment Tyrian purple, was studied theoretically at the TDDFT level of theory. We found that the absorption maximum of the compound about $600 \mathrm{~nm}$ underwent a red-shift when moving from the gas phase in solution. With the located two conical intersections $S_{0} / S_{1}$ we investigated (TD-DFT) the mechanisms connecting these structures with the ground state equilibrium geometry. It was established that the conical intersections are not accessible along any of the excited-state reaction paths which implies optical deactivation of the first excited state of the compound. With respect to these mechanisms the pigment should exhibit high photostability when exposed to visible light.

Keywords: conical intersections, excited states, linear interpolation in internal coordinates, Tyrian purple.

\section{INTRODUCTION}

$\mathbf{T}$ YRIAN purple is a pigment produced by some marine snails belonging to the family of Muricidae.[1] The pigment is produced as a colorless secretion in the hypobranchial gland and turns purple on exposure to light and air. ${ }^{[2]}$ The historian Caseau confessed that " 10,000 shellfish would produce 1 gram of dyestuff, and that would only dye the hem of a garment in a deep colour"..[3] The major component of the ancient dye Tyrian purple is the compound 6,6'-dibromoindigo (DBI).

Tyrian purple is the most famous dye in antiquity and during the Middle Ages which has been used by Egyptians and subsequently by Greeks and Romans. ${ }^{[1]}$ Byzantium emperors have used Tyrian purple as a symbol of power and royalty and they believe that it possesses supernatural and magic power. ${ }^{[3]}$ Tyrian purple has been adopted by the Romans as a symbol of imperial status and authority. The emperor's silk cloths must be dyed with Tyrian purple. Even more the Byzantium emperors were usually depicted (on mosaics and wall-paintings) wearing Tyrian purple cloths.

Tyrian purple has one unusual and valuable property: the colour keeps bright when exposed to sunlight, without fading, even hundreds of years after manufactur- ing of the dye. ${ }^{[5]}$ It could be a result of the high photostability of the compound 6,6'-dibromoindigo constituting the pigment. Unfortunately the photochemistry and the photophysics of the compound are not known so far.

Despite these facts it is known that the leuco forms of brominated indigoids can be debrominated by UV light. ${ }^{[6-8]}$ However this process is unspecific for DBI at room light. ${ }^{[6,9]}$

In the beginning of the past century Friedländer for the first time has proposed the composition of Tyrian purple to be $6,6^{\prime}$-dibromoindigo. ${ }^{[10]}$ Shortly after that the compound 6,6'-dibromoindigo and other indigo derivatives have been synthesized for the first time. ${ }^{[11]}$ Later Wolk et al have proposed a detailed scheme for the preparation of $6,6^{\prime}$-dibromoindigo from different reagents. ${ }^{[12,13]}$

In water solution 6,6'-dibromoindigo has a blue color $(590 \mathrm{~nm})$ whereas the color of dyed cloths (on wool) is purple $(520 \mathrm{~nm})$. $^{[5,6,14]}$ This unusual shift of the absorption maximum from $590 \mathrm{~nm}$ to $520 \mathrm{~nm}$ might be a result of conformational changes in the compound. Some authors have proposed the IR and RAMAN spectra of the compound as well as the X-ray crystal structure of the dye immobilized on wool. $[5,14,15]$ The experimental findings of Cooksey et al. ${ }^{[5,14]}$ have given an evidence only for one conformer of

(cc) Br 
6,6'-dibromoindigo. This is the planar conformer with the two carbonyl groups located on opposite sides of the molecule.

The crystal structure of 6,6'-dibromoindigo has been discussed in details by Truger ${ }^{[16]}$ However the first crystallographic study of the compound has been carried out several decades before that by Süsse et al..[17] and Larsen et al. ${ }^{[18]}$ They have proposed space group $\mathrm{P} 21 / \mathrm{a}$ and $\mathrm{P} 2_{1} / \mathrm{c}$. The study of Süsse et al.[17] have reported the next unit cell parameters $a=11.5 \AA, b=4.85 \AA, c=12.6 \AA, \beta=104.0^{\circ}$. The molecules in the unit cell form $\mathrm{H}$-bonds with four neighboring molecules and also form $\pi$-stacked aggregates. ${ }^{[16]}$

The aim of the research is to try to explain the high sunlight stability of the pigment Tyrian purple or the compound 6,6'-dibromoindigo (DBI). The investigation of the UV light phenomena is outside the scope of the current paper.

\section{THEORETICAL METHODS}

The ground-state equilibrium geometry of DBI was optimized at the BLYP/cc-pVDZ level of theory in the gas phase. Subsequent optimizations of the ground-state geometry of the compound were performed in water, acetonitrile and chloroform surroundings at the same level applying polarizable continuum model (PCM). The gas phase equilibrium geometry of DBI was also reoptimizaed at the CASSCF(2,2)/STO-3G* level of theory. At the same level we also found two conical intersections $S_{0} / S_{1}$. One of them is connected with a mutual orientation of the two 6bromoindigo rings while the second one is connected with a deviation of one of the bromine atoms out of the molecular plane. It should be mentioned that the attempts to enlarge the active space and to use better basis set failed because of the large size of the molecule and the presence of two bromines. The CASSCF structures were used only to perform linear interpolation in internal coordinates (LIIC).

The vertical excitation energies of DBI were calculated at the BLYP/cc-pVDZ level of theory with the ground-state equilibrium geometries found in different media (gas phase, water, acetonitrile and chloroform) with PCM. The accuracy of the TD-DFT methods has been discussed in lots of theoretical investigations and it has been demonstrated that they predict reliable excited state properties. ${ }^{[19-21]}$ It has been proven that the TD-DFT is one of the most powerful and accurate method for such explorations. ${ }^{[20]}$ For DBI the DFT method has predicted close results to the experimental UV absorption maxima. ${ }^{[22]}$ The accuracy of the BLYP functional was commented in our recent paper ${ }^{[23]}$ and we found that this functional predicts excitation energies close to the experimental UV absorption maxima of organic compounds in solvents.
In order to get the excited-state reaction paths of the mechanisms connecting the ground-state minimum of DBI with the two conical intersections $\mathrm{S}_{0} / \mathrm{S}_{1}$ we performed linear interpolation in internal coordinates. In this approach we used the CASSCF structures. The LIIC approach was performed by the linear equation:

$$
Q_{i}=Q_{i}(I)+\varepsilon\left[Q_{i}(E)-Q_{i}(I)\right]
$$

where $Q_{i}(I)$ is the $i$-th coordinate of the initial structure (ground state of $\mathrm{DBI}) ; \mathrm{Q}_{j}(\mathrm{E})$ is the same coordinate of the final structure (conical intersection $\mathrm{S}_{0} / \mathrm{S}_{1}$ ); $\varepsilon$ is the interpolation parameter, whose values are in the interval 0 (initial structure) $\div 1$ (final structure) and it is regarded as a reaction coordinate here. Between the initial and the final structure the LIIC approach led to several structures whose vertical excitation energies were computed at the TD BLYP/cc-pVDZ level.

The calculations were carried out with the GAUSSIAN 03 commercial program package ${ }^{[24]}$ and the structures were visualized with the ChemCraft program. ${ }^{[25]}$

\section{RESULTS AND DISCUSSION}

\section{Ground-State Equilibrium Geometry and Conical Intersections $S_{0} / S_{1}$ of DBI}

The gas-phase ground-state equilibrium geometry of $\mathrm{DBI}$ obtained at the BLYP level is presented in Figure 1a. As seen the molecule is planar and the two carbonyl groups are located on opposite sides of the molecule. We tried to optimize another planar conformer of DBI with two carbonyl groups located on one side of the molecule (S-cisconformer) but the calculations failed without locating a minimum. Perhaps it is due to the strong repulsion between the two oxygen atoms. The S-cis-conformer of DBI has been not reported in the available literature. ${ }^{[6,22]}$

The conical intersection $\mathbf{C l}_{\mathbf{A}}$ has a non-planar geometry (Figure 1b). It describes a mutual motion of the two aromatic fragments one to another. It could be associated with a rotation around the $\mathrm{C}_{16}=\mathrm{C}_{8}$ bond similar to ethylene. ${ }^{[26]}$ In other words so-called "ethylene-like" conical intersection is realized. ${ }^{[26,27]}$ As known the main deactivation channels of nucleic acid bases are connected with non-radiative excited-state deactivation through ethylenelike conical intersections $S_{0} / S_{1}$. ${ }^{[28-31]}$

The conical intersection $\mathrm{Cl}_{\mathrm{B}}$ shows a structure with one bromine atom out of the molecular plane: $\left\langle\mathrm{C}_{3} \mathrm{C}_{2} \mathrm{C}_{1} \mathrm{C}_{6}=\right.$ $68.6^{\circ} ;<\mathrm{C}_{1} \mathrm{C}_{2} \mathrm{C}_{23} \mathrm{C}_{4}=-68.6^{\circ} ;<\mathrm{Br}_{12} \mathrm{C}_{2} \mathrm{C}_{1} \mathrm{C}_{6}=-92.1^{\circ}$. Similar conical intersection has been found for 5 -fluorouracil[32] and it well describes the non-radiative deactivation of the 


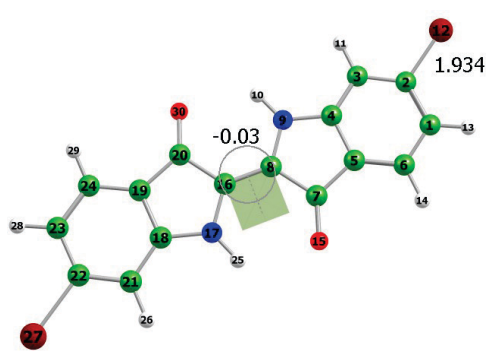

(a)

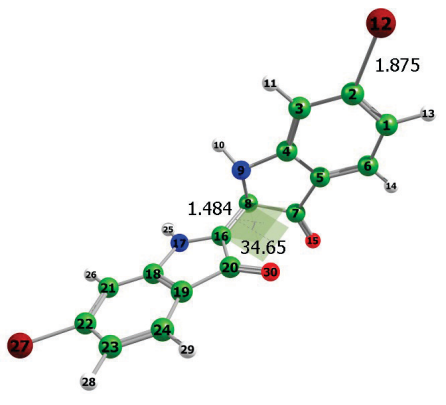

(b)

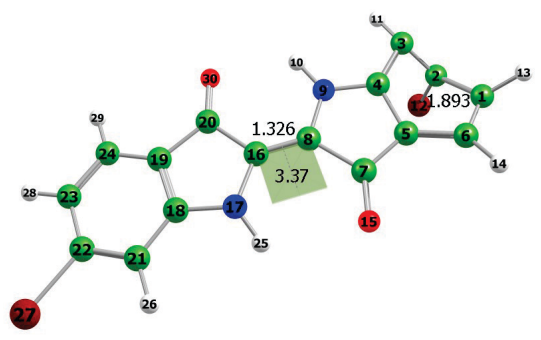

(c)

Figure 1. (a) BLYP-ground-state equilibrium geometry of DBI; (b) CASSCF-conical intersection $\mathrm{Cl}_{\mathrm{A}}$ of $\mathrm{DBI}$ connected with a $\mathrm{C}_{16} \mathrm{C}_{8}$ twist; (c) CASSCF-conical intersection $\mathrm{Cl}_{\mathrm{B}}$ connected with a deviation of one bromine atom out of the molecular plane.

compound. In fact this conical intersection $\mathrm{S}_{0} / \mathrm{S}_{1}$ is a crossing point between the ground state PES and the ${ }^{1} \pi \pi^{*} \mathrm{PES} .{ }^{[32]}$

\section{Vertical Excitation Energies}

The calculated vertical excitation energies of the groundstate equilibrium structure of $\mathrm{DBI}$ in different surroundings are given in Table 1. Selected molecular orbitals involved in the electron transitions are given in Figure 2. The lowestlying excited state in all phases is the spectroscopically active ${ }^{1} \pi \pi^{*}$ excited state. As seen the passage from the gas phase to solvent provokes a red-shift of the vertical excitation energy of the first bright excited state. This effect can be seen also from the simulated UV spectra in Figure 3 of $\mathbf{D B I}$ in the different phases. The dielectric properties of the solvents do not influence the vertical excitation energy of this excited state. The same effect has been observed by Sheppard et al.[33] for indigo in vacuum and non-polar solvent $\mathrm{CCl}_{4}$ and the polar solvent acetone. In these solvents the absorption maxima for the lowest-lying excited state were found to be at $2.271 \mathrm{eV}(546 \mathrm{~nm}), 2.066$ $\mathrm{eV}(600 \mathrm{~nm})$ and $2.077 \mathrm{eV}(597 \mathrm{~nm})$ respectively. This absorption for DBI has been registered at $2.073(598 \mathrm{~nm})$ eV in DMSO ${ }^{[22,34]}$ and methanol. [35] The bathochromic effect of indigoides, in this number DBI, has been also well documented in the work of Głowacki et al. ${ }^{[36]}$
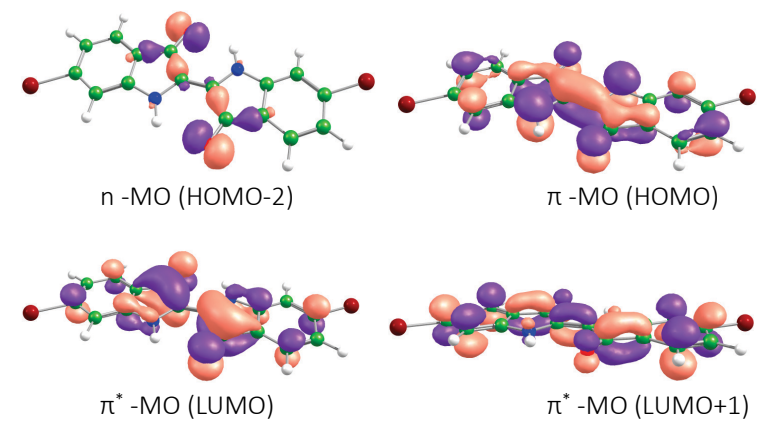

Figure 2. Selected BLYP-molecular orbitals in the gas phase, involved in the electron transitions.
The vertical excitation energies about $3.8 \mathrm{eV}$ correspond to the electron transition $\pi \rightarrow \pi^{*}$ for which we calculated the largest value of the oscillator strength in all phases. The values should correspond to the most intensive

Table 1. BLYP-vertical excitation energies (VEE) of DBI in different surroundings, all in $\mathrm{eV}$. The oscillator strength is given in brackets.

\begin{tabular}{|c|c|c|c|c|c|c|c|}
\hline \multicolumn{2}{|c|}{ Gas phase } & \multicolumn{2}{|c|}{ Water } & \multicolumn{2}{|c|}{ Acetonitrile } & \multicolumn{2}{|c|}{ Chloroform } \\
\hline State & VEE & State & VEE & State & VEE & State & VEE \\
\hline${ }^{1} \pi \pi^{*}$ & $\begin{array}{c}2.006 \\
(0.2543)\end{array}$ & ${ }^{1} \pi \pi^{*}$ & $\begin{array}{c}1.894 \\
(0.3204)\end{array}$ & ${ }^{1} \pi \pi^{*}$ & $\begin{array}{c}1.894 \\
(0.3219)\end{array}$ & ${ }^{1} \pi \pi^{*}$ & $\begin{array}{c}1.894 \\
(0.3349)\end{array}$ \\
\hline${ }^{1} n \pi^{*}$ & $\begin{array}{c}2.255 \\
(0.0)\end{array}$ & ${ }^{1} \pi \pi^{*}$ & $\begin{array}{c}2.216 \\
(0.0)\end{array}$ & ${ }^{1} \pi \pi^{*}$ & $\begin{array}{c}2.217 \\
(0.0)\end{array}$ & ${ }^{1} \pi \pi^{*}$ & $\begin{array}{c}2.228 \\
(0.0)\end{array}$ \\
\hline${ }^{1} \pi \pi^{*}$ & $\begin{array}{c}2.269 \\
(0.0)\end{array}$ & ${ }^{1} n \pi^{*}$ & $\begin{array}{c}2.288 \\
(0.0)\end{array}$ & ${ }^{1} n \pi^{*}$ & $\begin{array}{c}2.287 \\
(0.0)\end{array}$ & ${ }^{1} n \pi^{*}$ & $\begin{array}{c}2.278 \\
(0.0)\end{array}$ \\
\hline${ }^{1} n \pi^{*}$ & $\begin{array}{c}2.660 \\
(0.0)\end{array}$ & ${ }^{1} n \pi^{*}$ & $\begin{array}{c}2.695 \\
(0.0)\end{array}$ & ${ }^{1} n \pi^{*}$ & $\begin{array}{c}2.694 \\
(0.0)\end{array}$ & ${ }^{1} n \pi^{*}$ & $\begin{array}{c}2.685 \\
(0.0)\end{array}$ \\
\hline${ }^{1} \pi \pi^{*}$ & $\begin{array}{c}2.718 \\
(0.2287)\end{array}$ & ${ }^{1} \pi \pi^{*}$ & $\begin{array}{c}2.702 \\
(0.2784)\end{array}$ & ${ }^{1} \pi \pi^{*}$ & $\begin{array}{c}2.701 \\
(0.2798)\end{array}$ & ${ }^{1} \pi \pi^{*}$ & $\begin{array}{c}2.693 \\
(0.2913)\end{array}$ \\
\hline \multicolumn{8}{|c|}{---} \\
\hline${ }^{1} \pi \pi^{*}$ & $\begin{array}{c}3.894 \\
(0.5889)\end{array}$ & ${ }^{1} \pi \pi^{*}$ & $\begin{array}{c}3.858 \\
(0.7565)\end{array}$ & ${ }^{1} \pi \pi^{*}$ & $\begin{array}{c}3.857 \\
(0.7588)\end{array}$ & ${ }^{1} \pi \pi^{*}$ & $\begin{array}{c}3.848 \\
(0.7775)\end{array}$ \\
\hline
\end{tabular}

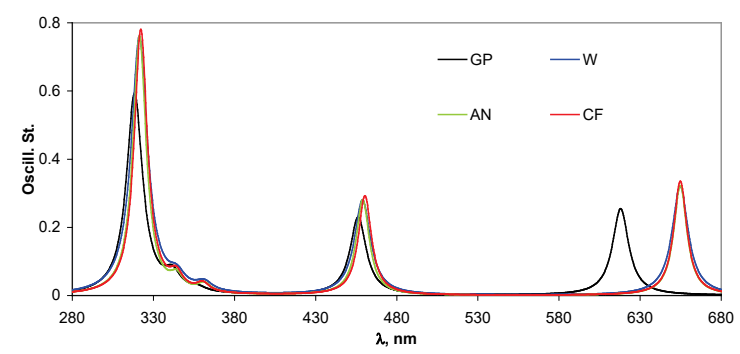

Figure 3. Theoretical (BLYP) UV absorption maxima of DBI in different phases (GP - gas phase; W - water; AN acetonitrile; CF - chloroform). The spectra were simulated using Lorenzian broadening of the bands. The groundstatate structures were optimized in the gas phase and in a given solvent (PCM). 
band in the experimental spectrum of DBI in solvents. The simulated bands are given in Figure 3. They show a slight solvatochromic effect: the decrease of the dielectric constant of the solvent leads to a hardly visible red-shift of the maximum.

The simulated bands in the middle interval of wavelengths $430-480 \mathrm{~nm}$ show the same trend as for the most intensive band. The bands correspond to the vertical excitation energies of $\mathrm{DBI}$ at about $2.7 \mathrm{eV}$ which we assigned to the ${ }^{1} \pi \pi^{*}$ excited states either.

\section{Excited-State Reaction Paths}

To check the high photostability of DBI when exposed to visible light we performed a linear interpolation in internal coordinates between the ground-state minimum and the conical intersections $S_{0} / S_{1}$. The BLYP-linearly interpolated excited-state reaction curves in the gas phase and water surroundings are presented in Figure 4 for the transformations $\mathrm{DBI} \rightarrow \mathrm{Cl}_{\mathbf{A}}$ and $\mathrm{DBI} \rightarrow \mathrm{Cl}_{\mathbf{B}}$. As seen in both cases the ${ }^{1} \pi \pi^{*}$ excited-state reaction paths show increase of energy along the reaction coordinate which is unusual behavior of this state as compared to the canonical nucleic acid bases and similar mechanisms.[28,32,37-39] In other words the conical intersections $\mathbf{C l}_{A}$ and $\mathbf{C l}_{B}$ are not accessible through the ${ }^{1} \pi \pi^{*}$ excited state reaction paths of the first bright excited state. Thus pumping this state by excitation with visible light cannot lead to some photophysical phenomena which are connected with the non-optical transitions through conical intersections, in particular the conical intersections $\mathrm{Cl}_{\mathbf{A}}$ and $\mathrm{Cl}_{\mathbf{B}}$. The excited DBI to the first ${ }^{1} \pi \pi^{*}$ excited state can only participate in optical transition (fluorescence) to the ground state in the Franck - Condon area. Non-accessibility of the two conical intersections prevents $\mathbf{D B I}$ to be transformed in another structure which would be responsible for the change of the color of DBI. The second ${ }^{1} \pi \pi^{*}$ and the dark ${ }^{1} n \pi^{*}$ excited-state reaction paths show also an energy increase along the reaction coordinate.

\section{CONCLUSION}

We performed a theoretical study of 6,6'-dibromoindigo at the TD-DFT level of theory. It is a component of the ancient dye Tyrian purple known as "imperial color". We studied the vertical excitation energies and the theoretical absorption spectra of the compound. It was found that the passage from the gas phase to solvents the lowest-lying ${ }^{1} \pi \pi^{*}$ excited state, which is the first spectroscopically active state, underwent a considerable red-shift in the visible part of the spectrum. Two conical intersections $S_{0} / S_{1}$ between the ground state and the lowest-lying excited state were also located. Their structures were used to perform linear interpolation in internal coordinates between the groundstate minimum of the compound and the conical intersections. The obtained excited-state reaction paths revealed that the conical intersections are not accessible through the energy curves of the lowest-lying excited state. In other words, with respect to these mechanisms, the main deactivation of the first excited state of 6,6'-dibromoindigo is an optical transition $S_{1} \rightarrow S_{0}$, which could explain the high photostability of the pigment when exposed to visible light.
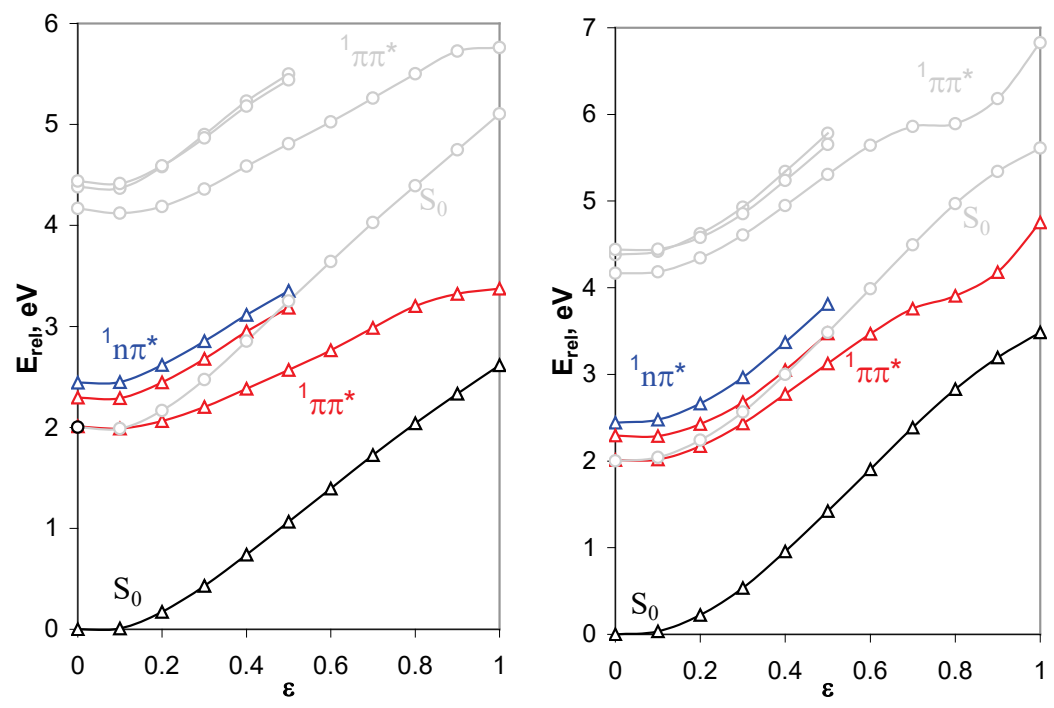

Figure 4. Linearly-interpolated excited-state reaction paths of the reactions a) $\mathrm{DBI} \rightarrow \mathrm{Cl}_{\mathrm{A}}$ and b) $\mathrm{DBI} \rightarrow \mathrm{Cl}_{\mathrm{B}}$. The triangles $(\Delta)$ describe the reaction curves for the mechanisms in water surroundings, while the circles (O) are for the gas-phase mechanism. The relative energy was calculated according to the energy of the ground-state equilibrium geometry in water surroundings (-6022.633987 a.u.). The $x$-axis is scaled according to the values of the interpolation parameter $\varepsilon$. 


\section{REFERENCES}

[1] L. C. A. Naegel, C. J. Cooksey, J. of Shellfish Res. 2002, 21, 193-200.

[2] V. Fretter, A. Graham: British Prosobranch Molluscs. Their functional anatomy and ecology (Revised and updated edition), The Ray Society, London, 1994.

[3] R. Bagnall et al.: The Encyclopedia of Ancient History, Wiley-Blackwell, 2012.

[4] M. Reinhold, Collection Latomus 1970, 116, 5-73.

[5] C. J. Cooksey, Molecules 2001,6, 736-769. https://doi.org/10.3390/60900736

[6] O. Lavinda, I. Mironova, S. Karimi, F. Pozzi, J. Samson, H. Ajiki, L. Massa, K. Ramig, Dyes and Pigments 2013 , 96, 581-589.

https://doi.org/10.1016/j.dyepig.2012.07.014

[7] J. van Alphen, Rec. Trav. Chim. Pays-Bas. 1944, 63, 95-96.

[8] R. C. Hoffman, R. C. Zilber, R. E. Hoffman, Magn. Res. Chem. 2010, 48, 892-895.

https://doi.org/10.1002/mrc.2685

[9] Z. C. Koren, J. Soc. Dyers. Colourists 1994, 110, 273-277. https://doi.org/10.1111/j.1478-4408.1994.tb01656.x

[10] P. Friedländer, Z. Angew. Chem. 1909, 22, 23212324. https://doi.org/10.1002/ange.19090224802

[11] P. Friedländer, S. Bruckner, G. Deutsch, Justus Liebig's Annalen der Chemie 1912, 388, 23-49. https://doi.org/10.1002/jlac.19123880103

[12] J. L. Wolk, A. A. Frimer, Molecules 2010, 15, 54735508. https://doi.org/10.3390/molecules15085473

[13] J. L. Wolk, A. A. Frimer, A Simple, Molecules 2010, 15, 5561-5580.

https://doi.org/10.3390/molecules15085561

[14] R. J. H. Clark, C. J. Cooksey, New J. Chem. 1999, 23, 323-328. https://doi.org/10.1039/a808562e

[15] K. Ramig, O. Lavinda, D. J. Szalda, I. Mironova, S. Karimi, F. Pozzi, N. Shah, J. Samson, H. Ajiki, L. Massa, D. Mantzouris, I. Karapanagiotis, C. Cooksey, Dyes and Pigments 2015, 117, 37-48.

https://doi.org/10.1016/j.dyepig.2015.01.025

[16] M. Truger, Master's thesis, Graz University of Technology, 2015.

[17] P. Süsse, C. Krampe, Naturwissenschaften 1979, 66, 110. https://doi.org/10.1007/BF00373505

[18] S. Larsen and F. Watjen, Acta Chem. Scand A 1980, 34, 171-176.

https://doi.org/10.3891/acta.chem.scand.34a-0171

[19] D. Jacquemin, E.A. Perpete, I. Ciofini, C. Adamo, J. Chem. Theory Comput. 2010, 6, 1532-1537. https://doi.org/10.1021/ct100005d

[20] C. Adamo, D. Jacquemin, Chem. Soc. Rev. 2013, 42, 845-856. https://doi.org/10.1039/C2CS35394F
[21] E. A. Perpete, V. Wathelet, J. Preat, C. Lambert, D. Jacquemin, J. Chem. Theory Comput. 2006, 2, 434440. https://doi.org/10.1021/ct050287w

[22] G. Verri, C. M. de Fonjaudran, A. Acocella, G. Accorsi, D. Comelli, C. D'Andrea, A. Nevin, F. Zerbetto, D. Saunders, Dyes and Pigments 2019, 160, 879-889. https://doi.org/10.1016/j.dyepig.2018.08.027

[23] I. G. Shterev, V. B. Delchev, Spectrochimica Acta Part A 2014, 125, 384-390.

https://doi.org/10.1016/j.saa.2014.01.124

[24] M. J. Frisch, G. W. Trucks, H. B. Schlegel, G. E. Scuseria, M. A. Robb, J. R. Cheeseman, J. A. Montgomery, Jr., T. Vreven, K. N. Kudin, J. C. Burant, J. M. Millam, S. S. Iyengar, J. Tomasi, V. Barone, B. Mennucci, M. Cossi, G. Scalmani, N. Rega, G. A. Petersson, H. Nakatsuji, M. Hada, M. Ehara, K. Toyota, R. Fukuda, J. Hasegawa, M. Ishida, T. Nakajima, Y. Honda, O. Kitao, H. Nakai, M. Klene, X. Li, J. E. Knox, H. P. Hratchian, J. B. Cross, V. Bakken, C. Adamo, J. Jaramillo, R. Gomperts, R. E. Stratmann, O. Yazyev, A. J. Austin, R. Cammi, C. Pomelli, J. W. Ochterski, P. Y. Ayala, K. Morokuma, G. A. Voth, P. Salvador, J. J. Dannenberg, V. G. Zakrzewski, S. Dapprich, A. D. Daniels, M. C. Strain, O. Farkas, D. K. Malick, A. D. Rabuck, K. Raghavachari, J. B. Foresman, J. V. Ortiz, Q. Cui, A. G. Baboul, S. Clifford, J. Cioslowski, B. B. Stefanov, G. Liu, A. Liashenko, P. Piskorz, I. Komaromi, R. L. Martin, D. J. Fox, T. Keith, M. A. Al-Laham, C. Y. Peng, A. Nanayakkara, M. Challacombe, P. M. W. Gill, B. Johnson, W. Chen, M. W. Wong, C. Gonzalez, and J. A. Pople, Gaussian 03, Revision D.01, Gaussian, Inc., Wallingford CT, 2004.

[25] G. A. Andrienko: ChemCraft version 1.8 (Build 523a)

[26] M. Barbatti, J. P., H. Lischka, J. Chem. Phys. 2004, 121, 11614-11624.

https://doi.org/10.1063/1.1807378

[27] N. Minezawa, M. S. Gordon, J. Phys. Chem. A 2009, 113, 12749-12753. https://doi.org/10.1021/jp908032x

[28] W. Domcke, D. R. Yarkony: Conical Intersections, World Scientific, Singapore, 2004. https://doi.org/10.1142/5406

[29] L. Serrano-Andres, M. Merchan, in Radiation Induced Molecular Phenomena in Nucleic acids (Eds. M. K. Shukla, K. Leszczynski), Springer, 2008.

[30] S. Matsika, J. Phys. Chem. A 2004, 108, 7584-7590. https://doi.org/10.1021/jp048284n

[31] S. Matsika, J. Phys. Chem. A 2005, 109, 7538-7545. https://doi.org/10.1021/jp0513622

[32] S. Yamazaki, T. Taketsugu, J. Phys. Chem. A 2012, 116, 491-503. https://doi.org/10.1021/jp206546g

[33] S. E. Sheppard, P. T. Newsome, J. Am. Chem. Soc. 1942, 64, 2937-2946. https://doi.org/10.1021/ja01264a059 
[34] Z. C. Koren, Mater. Res. Soc. Symp. Proc. 2012, 1374, 29-48. https://doi.org/10.1557/opl.2012.1376

[35] Z. C. Koren in Indirubin, the red shade of indigo (Eds. L. Meijer, N. Guyard, L. Skaltsounis, G. Eisenbrand), Roscoff: Life in Progress Editions; 2006.

[36] E. D. Głowacki, G. Voss, N. S. Sariciftci, Adv. Mater. 2013, 25, 6783-6800.

https://doi.org/10.1002/adma.201302652
[37] V. B. Delchev, W. Domcke, J. Photochem. Photobiol. A: Chemistry 2013, 271, 1-7.

[38] P. B. Kancheva, V. B. Delchev, J. Mol. Mod. 2016, 22, 230.

https://doi.org/10.1007/s00894-016-3087-9

[39] V. B. Delchev, E. Horkel, D. Svatunek, J. Mol. Struct. 2020, 1205, 127571(8).

https://doi.org/10.1016/j.molstruc.2019.127571 